\title{
Einleitung: Das Unbehagen an der Technik und die Macht des Rechts
}

Die Ausgangslage und die Gegenstände der Diskussion

Dem Internet werden Wirkungen vielfältigster Art zugeschrieben - Veränderungen der sozialen Welt, der Politik, der Wirtschaft und der individuellen Lebensverhältnisse. Das Internet, so heißt es, schafft ganz neue Freiheit(en), erweitert unseren Lebensraum und unser Wissen, vermittelt uns Zugang zu anderen Kulturen, lässt uns am Leben anderer teilnehmen und neue Freundschaften knüpfen, eröffnet neue Geschäftsfelder, formt die politische Willensbildung auf allen Ebenen neu, stärkt also die Demokratie. „Das Internet prägt unser Leben, ob wir wollen oder nicht, ob wir mitmachen oder nicht. Gesellschaft und Wirtschaft funktionieren nicht mehr ohne. "5 Überall liest man inzwischen, wir lebten in einer „,digitalen Gesellschaft" oder einer „digitalen Demokratie“.

Die Bewusstseinslage ist überdies dadurch kompliziert, dass die einen die Technik als Heilsbringer ansehen, während andere sie als fundamentale Bedrohung unserer Kultur betrachten. Es ist zwar stark übertrieben, einen „digitalen Bürgerkrieg“ zu diagnostizieren, aber von Einigkeit über Segen oder Fluch der Technik sind wir weit entfernt, und es ist nicht abwegig, von einem Zusammenstoß der Kulturen zu sprechen.

Wie ist die Lage wirklich? Was ist damit gemeint, dass ,unser Leben“ durch das Internet ,geprägt“ werde und dass Gesellschaft und Wirtschaft ohne dieses nicht mehr „funktionieren“? Immerhin haben alle früheren Generationen ohne ein elektronisches Netz kommuniziert, und die sozialen Systeme haben auch damals „,funktioniert“. Aus dieser vermeintlich grauen Vorzeit sind uns geistige Schätze überliefert, die nach wie vor die wesentlichen Inhalte aller kulturellen Produktion und Konsumtion ausmachen und neue Kreation anregen. Wer das Internet zum Schlüssel aller gesellschaftlichen Verhältnisse und kultureller Prägung erklärt, hat eine zu enge Perspektive. Gewiss sind Verwaltung und industrielle Produktion von zuverlässiger Informationsverarbeitung abhängig, aber die Lebenssituation der meisten Menschen und die Verteilung von Macht und Wohlstand werden davon nur mittelbar beeinflusst. Wesentlich für den Zustand der Gesellschaft sind ganz andere

5 Fischermann/Hamann 2011, S. 12. Wie das Internet „unser Leben“ und „unser Denken“ „prägt“, wird in zahlreichen Publikationen der letzten Jahre behauptet und mit Beispielen illustriert. Ein Beispiel für alle: Görig 2011. Die Süddeutsche Zeitung bringt u.v.a. eine Doppelseite mit Beiträgen unter dem Titel „Gefangen im Netz“(17./18.3.2012). Miriam Meckel fragt: „Wie lange unterscheiden wir uns noch vom Computer?“ (Meckel 2012). 
Faktoren: die globalen Machtstrukturen, der Zustand der natürlichen Umwelt, die wirtschaftliche Entwicklung der einzelnen Länder, die Arbeitsbedingungen und die Einkommens- und Vermögensverteilung, das Wohnumfeld, die Verkehrsprobleme - und für den Einzelnen nicht zuletzt die unmittelbaren persönlichen Beziehungen $\mathrm{zu}$ anderen Menschen.

Die Rede von der „digitalen Gesellschaft“ und der ,digitalen Demokratie“ führt in die Irre. Denn diese Begriffe verweisen ja nicht auf soziale Strukturen, sie bezeichnen vielmehr nur den Umstand, dass wir uns der ,digitalen“ Technik bedienen. Sprachlich ist auch dies fragwürdig. „Digital“ bedeutet „ziffernmäßig“ und verweist auf die Besonderheit der elektronischen Prozesse, dass sie auf der Umsetzung aller anderen Signale in die Ziffern 0 oder 1 beruhen. Dieses Charakteristikum der elektronischen Informationsverarbeitung ist in der Außenwelt, in den Wirkungen der technischen Prozesse nicht erkennbar und hat keine Wirkungen. Wer ständig auf einen Bildschirm schaut, seine Informationen überwiegend aus dem Netz bezieht und seine Kommunikation überwiegend über das Netz laufen lässt, mag sich als „homo reticuli“ fühlen. ${ }^{6}$ Die meisten Menschen aber leben und denken nicht „digital“, sondern nach wie vor als soziale Wesen unter anderen. So halten die Bürger insgesamt ,an ihren eingefahrenen Routinen der politischen Kommunikation“ fest. ${ }^{7}$ Richtiger wäre es, von „Informationsgesellschaft“ oder vielmehr „Informationstechnikgesellschaft“ zu sprechen. Auch der Ausdruck „Netzwerkgesellschaft" ${ }^{48}$ ist passender als ,Informationsgesellschaft".

Betrachten wir also das Internet und seine Wirkungen genauer, ohne auf die übersteigerten und irritierenden Vorstellungen der unkritisch Faszinierten hereinzufallen, und zwar zunächst von außen: Wie nutzen wir es? Welche Wirkungen treten ein? ${ }^{9}$ Auf diesem Wege können wir eine genauere Vorstellung davon erlangen, welche Vorgänge überhaupt als rechtlich regelungsbedürftig in Betracht kommen. Erst wenn hierüber Konsens besteht, ist es sinnvoll, andere Ansätze - also politische, mediale, pädagogische, ökonomische oder sonstige Einwirkungen auf das Verhalten der Menschen - zu erörtern.

6 Übersetzt also: „Netzmensch“, gemeint ist „vernetzter Mensch“; genauer wäre: Mensch des kleinen Netzes, so Kurz/Rieger 2012, S. 7.

7 Emmer/Vowe/Wolling 2011, S. 308.

8 Ihn benutzt Sieber 2012, S. 10.

9 Sehr interessante empirisch begründete Aussagen zur Entwicklung der politischen OnlineKommunikation in Deutschland enthält die Studie von Emmer/Vowe/Wolling: „Bürger online“(2011). 


\section{Die Vielfalt der Nutzungen}

Beim Surfen im Internet kann man ins Schwärmen geraten. Was wir früher mit großer Mühe in vielen Büchern nachschlagen mussten, kommt heute sofort auf unsere Bildschirme. Wir können uns über die verschiedensten Themen schneller und umfassender informieren als jemals zuvor. Wir vergewissern uns vieler Dinge, die das Gedächtnis unscharf überliefert hat oder über die wir in den ,alten“ Medien zu wenig finden. Wir erhalten unerwartete Informationen, die unseren Horizont bereichern. Texte aus aller Welt, an die wir früher gar nicht herangekommen sind, werden sekundenschnell greifbar. Wir können uns am Computer hervorragend unterhalten - mit Filmen und Videos aller Art, politischen und unpolitischen Texten und Bildern, mit Geschichte und Geschichten, Science-fiction und Belletristik, und wir können Musik hören und Spiele spielen. Ein phantastischer Komfort!

Auch die E-Mail-Technik ist begeisternd. Ohne sie hätte ich manche Arbeit nicht oder nur viel langsamer fertigstellen können. Ich kann ohne Aufwand eine große Zahl von Empfängern ansprechen, mich in kurzem Takt mit anderen austauschen und streiten. So wird aus einseitiger Information eine mehrseitige Kommunikation. Sogar mit denen, die mir bisher nur etwas gesendet haben, kann ich kommunizieren: Die Rundfunkveranstalter ermöglichen zunehmend (und gewiss künftig noch wesentlich mehr) ihren Rezipienten, aus der passiven Konsumentenrolle zu schlüpfen und sich zu den Sendungen zu äußern, ja daran teilzunehmen.

Viele Millionen Menschen haben sich in „,sozialen Netzwerken“ eingeschrieben, um mit anderen noch mehr zu kommunizieren. Im „Facebook“ nennen sich die auf diese Weise zunächst technisch miteinander Verbundenen „Freunde“, im „Twitter“ heißen sie „Followers“. Ich habe mir Freunde und Korrespondenzpartner auf andere Weise gesucht und brauche die technikgestützten Netzwerke nicht, aber für unzählige Jüngere sind sie zu einem unverzichtbaren Teil ihres Lebens geworden.

Der größte Teil der Menschen in Deutschland hat bereits jetzt die Möglichkeit, PC und Internet zu nutzen und sich an ihren Möglichkeiten zu erfreuen. Die Zahl der Anschlüsse wächst ständig; nach neueren Angaben sind schon vier von fünf Deutschen regelmäßig online, bei den unter 30-jährigen sogar fast alle (98 Prozent $)^{10}$. Mitglieder in sozialen Netzwerken sind angeblich schon vierzig Millionen Deutsche. Die Nachrichten, „tweets“, „posts“ und wie immer auch die Produkte heißen, sind nicht mehr zählbar; die Schätzungen sind umwerfend, unvorstellbar. Die Unternehmen, die der Allgemeinheit diese Technik zur Verfügung stellen,

10 Davon geht z.B. die Grundlagenstudie „Vertrauen und Sicherheit im Internet“ aus, die das Sinus-Institut Heidelberg im Auftrag des Deutschen Instituts für Vertrauen und Sicherheit im Internet angefertigt hat (DIVSI 2011, S. 7). Weitere Angaben (u.a. vom BITKOM-Verband) bei Spindler 2012, S. 15 f. mit Fn. 43 f.; ausführliche Analyse der verschiedenen Formen von Mediennutzung auf der Grundlage der ARD/ZDF-Online-Studien: von Eimeren/ Frees 2012. S.a. www.ard-zdf-onlinestudie.de. 
werden unglaublich reich; sie speichern unbeschreibbar große Datenmengen und werten sie aus, angeblich zum Nutzen der Mitglieder, auf jeden Fall aber zum Nutzen der Werbung treibenden Wirtschaft und damit zu ihrem eigenen Nutzen.

Die Zahl der Internetanschlüsse besagt aber wenig über Art und Intensität der Internetnutzung und damit über die Dimensionen der abzuwehrenden Risiken. Ein großer Teil der gemeldeten Nutzer verhält sich keineswegs so aktiv wie die Fans, die ständig im Netz surfen und alles ausprobieren wollen, was angeboten wird. Internet ist ein ,Aktivitätsmedium“, ${ }^{11}$ aber sehr viele bleiben passiv, manche davon weil es ihnen schwerfällt, sich im Netz zu orientieren, oder weil sie den Zeitaufwand scheuen. Andere sind zwar aktiv, aber streben nicht nach neuen Erkenntnissen und erst recht nicht nach politischer Mitwirkung, sondern wollen sich informieren, Waren und Leistungen bestellen und sich unterhalten, z.B. Videos ansehen oder die Mediatheken der Fernsehsender besuchen. ${ }^{12}$ Die Idealisten, die andere Prioritäten setzen, schließen von sich auf alle anderen und schaffen sich damit ein falsches Bild von der Lebenswirklichkeit der Vielen. Allgemeine Regeln sind auf die normalen, typischen Fälle abzustellen; Ungewöhnliches, Besonderes kann und muss bei der Rechtsanwendung im Einzelfall berücksichtigt werden.

\section{Die Problemfelder im Einzelnen: Individualrechte und Allgemeininteressen}

Vor diesem Hintergrund sind es bestimmte Risiken der Techniknutzung, die rechtspolitisch vorrangig behandelt werden müssen. Es sind diejenigen Phänomene, die den Betroffenen Angst machen oder von Experten als bedrohlich bezeichnet werden (was nicht unbedingt dasselbe ist). Dabei sollten wir sowohl die Bedrohung von Individualrechten wie die Gefahren für das Allgemeinwohl betrachten. Die Einzelnen fürchten vor allem um die Bewahrung ihrer Privatsphäre und ihrer Geheimnisse und um die Sicherheit und Unbefangenheit der Kommunikation. Sie wollen nicht beobachtet werden, und sie wollen ihre Entscheidungsfreiheit behalten. Es geht um die Unbefangenheit der Kommunikation, um die Abwehr von Überwachung, „Verdatung“ und Manipulation. Mit der Entstehung des weltweiten Netzes ist das Interesse der Nutzer hinzugekommen, sich im Netz frei zu „bewegen“, sich überall zu informieren, alle gespeicherten Inhalte ungehindert und möglichst unentgeltlich zur Kenntnis zu nehmen und die eigenen Botschaften ungehindert zu verbreiten. Dabei entstehen Konflikte mit anderen, die das Gleiche wollen oder aber gerade nicht wollen, dass Dritte ihnen ,ins Gehege kommen“.

11 Eisel 2011, S. 41.

12 Einzelheiten des Nutzungsverhaltens u.a. bei von Eimeren/Frees 2012. 
Richtig verstandene Netzpolitik greift darüber weit hinaus. ${ }^{13}$ Der Staat hat nicht nur Freiheitssphären zu gewährleisten, sondern auch aktiv zum Schutz von Individual- und Gemeinschaftsgütern tätig zu werden. So muss er grundsätzlich (wenn auch nicht immer) gegen illegale Äußerungen vorgehen, strafbare Handlungen bekämpfen (Kinderpornographie ist nur ein Bereich von vielen!), und ganz allgemein Rechtsschutz zur Verfügung stellen, damit der Einzelne sich erfolgreich wehren kann, wenn er von anderen angegriffen, betrogen oder beraubt wird. Er muss einen Grundbestand an Kommunikations-Infrastruktur für die ganze Bevölkerung garantieren und unter Umständen durch Subventionen oder in eigener (bzw. kommunaler) Regie dafür sorgen, dass auch entlegene Teile des Landes an das weltweite Netz angeschlossen werden. Zugangsbarrieren sollen abgebaut werden, so dass jeder und jede sich im Internet informieren und äußern kann. In diesen $\mathrm{Zu}$ sammenhang gehören auch die Stichworte Netzneutralität und Freiheit der Internetdienste.

Netzpolitik will und muss sich schließlich auch darum kümmern, wie sich die Technisierung auf den Zustand der Gesellschaft auswirkt: Wie verteidigen und entwickeln wir die demokratische Teilhabe und die Grundwerte unserer Kultur, wie sorgen wir für die Bildung der nachwachsenden Generationen? Auch hinter diesen Fragen stehen Konflikte, nämlich Meinungs- und Interessenunterschiede über die richtige Entwicklung des Gemeinwesens. Es geht um Bildung und Ausbildung, um Medienpädagogik und Kulturpolitik. Von rechtlichen Regelungen und ihrer Durchsetzung wird hier niemand allzu viel erwarten; aber ganz unwichtig sind Normen auch auf diesen Feldern nicht.

Dem entsprechen die wichtigsten Themen, die sich bei der praktisch-politischen Bewältigung der Probleme des Internets stellen:

- Internetfreiheit: Gibt es ein „Recht auf Internet“ und was bedeutet es?

- Wirksamer Schutz des Persönlichkeitsrechts und anderer Individualrechte

- Sicherung und Ausgestaltung der Informationsfreiheit

- Urheber- und Leistungsschutzrechte versus Freiheit der Internetnutzung

- Effektuierung des Verbraucherschutzes

- Ausbau der Infrastruktur und Gleichbehandlung bei der Nutzung der Netze

Die zentralen Themen der Netzpolitik werden in den drei Hauptteilen dieser Schrift behandelt: im I. Teil die Rechtsstellung des Individuums (anknüpfend an die drei ersten Punkte der obigen Zusammenstellung), im II. Teil die ökonomische und technische Sicht auf die Konflikte im Internet (insbesondere Urheberrechtsfrage und Verbraucherschutz) und schließlich im III. Teil die - von den Individualrechtsfragen zu trennende - Diskussion über neue Formen der Demokratie. Zu kurz kommen dabei die kultur- und bildungspolitischen und -philosophischen Fragen;

13 Vgl. nur Holznagel/Schumacher 2011. 
sie sind hintergründig bedeutsam, aber nicht mehr Gegenstand dieser Schrift. Der IV. Teil befasst sich mit den rechtspolitischen Konsequenzen, also dem, was Netzpolitik bewirken sollte.

Zuvor aber ist zu erörtern, inwieweit Gesetzgebung und Rechtspraxis dazu beitragen können, die Entwicklung in die gewünschte Richtung zu lenken.

Was kann das Recht bewirken?

Wir können uns von der vermeintlichen Macht der Computer befreien - nicht als Maschinenstürmer wie seinerzeit die von der Industrie verdrängten Weber, sondern zum einen durch klugen Umgang mit den neuen Instrumenten, ${ }^{14}$ zum anderen indem wir die Menschen, die über die Apparate verfügen, bei deren Verwendung kontrollieren. Eben dies geschieht durch Recht und seine Durchsetzung.

\section{Maßstäbe setzen}

Freilich scheinen immer mehr Menschen dem Gesetzgeber zu misstrauen. Nicht nur dass sie von der Politik pauschal nichts Gutes erwarten - sie zweifeln auch an der Wirksamkeit von Rechtsnormen. Dazu besteht auch Anlass; in vieler Hinsicht lässt sich eine Erosion des Rechtsbewusstseins, ein Nachlassen der Rechtsbefolgung beobachten - keineswegs nur im Internet. Gleichzeitig wird jedoch gefordert: „Die Politiker müssen das Netz beherrschen, sonst beherrscht das Netz die Politik“15. „Die Politiker“ - das sind die Menschen, die für die Gesetzgebung verantwortlich sind. Gegen alle Politikverdrossenheit und Erosion des Rechts fordert also die Öffentlichkeit - mit Recht -, dass die Entwicklung durch Rechtsnormen gesteuert (oder zumindest beeinflusst) wird. Das Vertrauen in Recht und Staat ist zwar zurückgegangen, und auch die Mitglieder der Zivilgesellschaft vertrauen sich gegenseitig immer weniger. Aber das Vertrauen in die Akteure auf den verschiedenen Ebenen kann durch neues Recht oder die Wiederbelebung alter Rechtsprinzipien wieder gestärkt werden.

Das Recht bewährt sich nicht nur wenn es ,durchgesetzt“ wird. Es wirkt selbst dann, wenn viele es nicht befolgen. In Rechtsnormen werden zunächst einmal Maßstäbe gesetzt, aus denen Verhaltensregeln für Individuen und Staatsorgane und Beurteilungskriterien für tatsächliche Vorgänge und Zustände hergeleitet werden.

14 Dazu etwa Kurz/Rieger 2012, insbes. S. 247 ff. Ebenso in der Grundlinie Beckedahl/ Lüke 2012.

15 Wefing 2011. Dort auch die weiteren Zitate. In Pham/Wefing 2012 wird jedoch Entwarnung gegeben. 
Manchmal bleiben sie latent, aber unter veränderten Umständen werden sie manifest und verändern die Wirklichkeit.

\section{Die Ordnung der Werte}

In der Internet- und Computer-Diskussion lässt sich beobachten, dass zwar viel (und oft unkritisch) über die neuen Möglichkeiten gesprochen wird, die sich bieten, aber wenig von den Werten, deren Verwirklichung man anstrebt. Zwar werden ständig die Grund- und Menschenrechte und die Grundprinzipien der Demokratie beschworen, aber diese Bezugnahme bleibt meist abstrakt, und zu selten wird gefragt, wie diese hohen verfassungsrechtlichen Werte sich zu anderen Wertvorstellungen verhalten, die möglicherweise damit kollidieren könnten. Es fehlt an einer soliden Werteskala, an einer Rangordnung der Rechtspositionen, die bei genauer Betrachtung der Problembereiche in den Blick kommen. Gewiss ist es überaus schwierig, eine solche Werteordnung festzustellen, aber wenn ein konkreter Fall zu lösen ist, muss eben dies zwingend geschehen: Die eine Position muss - nach sorgfältiger Abwägung! - hinter der anderen zurückstehen. Typisch für viele aktuelle Diskussionen ist aber, dass Konflikte nicht eindeutig entschieden werden, dass man beide Seiten zufrieden stellen oder zumindest beschwichtigen möchte. Das heißt den Kuchen essen und ihn doch behalten wollen - ein allzu beliebtes Spiel nicht nur in der Politik.

Rechtspolitik muss Prioritäten bestimmen, um die stets knappen Ressourcen effektiv und effizient einzusetzen. Wenn für die Lebensmittelkontrolle nicht genügend Geld und Stellen zur Verfügung stehen, kann nicht gleichzeitig mehr Personal für den Schutz von Kundendaten verwendet werden. Wenn die Jugendämter unterbesetzt sind und deshalb potentielle Pflegeeltern nicht auf ihre Eignung prüfen können - wie es offenbar mehrfach geschehen ist; dadurch sind mehrere Kinder ums Leben gekommen -, dann verliert etwa die Forderung nach flächendeckenden Breitbandnetzen an Bedeutung. So wichtig Themen wie die Netzneutralität und die Transparenz der Datenverarbeitung auch sind - es gibt noch wichtigere, und manche hierzulande erhobenen Forderungen nach „mehr Datenschutz“ müssen den Bürgern ärmerer Staaten als sehr fernliegend und typisch für eine Luxusgesellschaft erscheinen.

Um noch konkreter zu werden: Wir sollten uns zum Beispiel dazu bekennen, dass Frieden, gewaltfreie Streitlösung den Vorrang vor der individuellen Handlungsfreiheit haben muss, dass soziale Gerechtigkeit vor der Gewinnmaximierung Einzelner rangiert, dass Strafverfolgung und Gefahrenabwehr durch Polizei und Justiz grundsätzlich (also mit Ausnahmen) höherrangig sind als der Persönlichkeitsschutz durch das Steuer- oder Bankgeheimnis und dass das Sozialgeheimnis, 
auf das sich Sozialarbeiter berufen, in manchen Situationen hinter dem Wohl eines Kindes zurückstehen muss. So wie es eine Sozialpflichtigkeit des Eigentums gibt, gibt es auch eine soziale Einbindung des Persönlichkeitsrechts. Dementsprechend muss der Aufwand, den der Staat und die Unternehmen betreiben, um persönliche Daten vor Ausspähung zu schützen, in einem angemessenen Verhältnis zu den Mitteln stehen, die für die Erfüllung anderer Pflichten verfügbar sind.

\section{Wertungswidersprüche und -unsicherheiten}

Weil über dergleichen Fragen zu wenig nachgedacht wird, begegnen wir in der öffentlichen Diskussion wie auch im Handeln von Staat und Wirtschaft irritierenden Widersprüchen und Unsicherheiten der Bewertung: Technische Innovationen wie die genaue Energieverbrauchsmessung, die einerseits im Zuge der Energiewende als große Fortschritte gefeiert werden, erscheinen andererseits als Teufelszeug, dessen Realisierung möglichst verboten oder zumindest streng eingeschränkt werden soll. So liest man derzeit in den Fachzeitschriften und den Tätigkeitsberichten der Datenschutzbeauftragten lange Artikel ${ }^{16}$ über das Smart Metering, also die Technik der detaillierten, auf die einzelnen Geräte bezogenen Messung des Energieverbrauchs und der Nutzungszeiten: Diese Methode berge „ein hohes Ausforschungspotential hinsichtlich der Lebensgewohnheiten der Betroffenen ". ${ }^{17}$ Nützliche, bisher als bürgerfreundlicher Service angesehene Auskünfte der Verwaltung - wie die Melderegisterauskünfte an Private - werden plötzlich als ,gesetzlicher Wahnsinn" ${ }^{18}$ bezeichnet - als wäre der Zweck, bei Postsendungen Adressänderungen zu berücksichtigen, vollkommen abwegig. ${ }^{19}$ Viele halten es für unerträglich, dass Wirtschaftsunternehmen potentiellen Kunden namentlich adressierte Werbung ins Haus schicken; der Nutzen der Werbung wird gering geschätzt. Selbst die Spendenwerbung für gemeinnützige Organisationen gilt manchen schon als rechtswidrige Handlung, wenn dabei Anschriften verwendet werden, die nicht ausdrücklich für diesen Zweck erlangt worden sind. Sogar die Werbung für Organspenden wird blockiert, weil angeblich schon eine gesetzliche Pflicht, sich zur eigenen Spendenbereitschaft zu äußern, ,rechtlich, vor allem datenschutzrechtlich bedenklich" sei. ${ }^{20}$ Die Freiheit der einen, auf die Frage nach der Spendenbereit-

16 Ein Beispiel: Hornung/Fuchs 2012 (mit zahlreichen weiteren Nachweisen ähnlicher Abhandlungen).

17 Entschließung der 80. Konferenz der Datenschutzbeauftragten, in: BfDI 2011, S. 57 f. Näheres dazu unten S. $59 \mathrm{f}$.

18 Bericht von Heribert Prantl über eine Äußerung des schleswig-holsteinischen Datenschutzbeauftragten Thilo Weichert, Süddeutsche Zeitung v. 7./8. 7. 2012.

19 Aus der Berichterstattung und Kommentierung der Medien sei nur auf Blechschmidt/Käppner 2012 und Esslinger 2012 verwiesen.

20 So der CDU-Bundestagsabgeordnete Jens Spahn laut Süddeutscher Zeitung vom 25.10.2011. 
schaft nicht zu antworten, wird also höher bewertet als das Ziel, zur Hilfe für schwerstkranke Menschen beizutragen. Über derartige sozial-ethische Kontroversen muss diskutiert werden, und die Entscheidungen müssen in den Formen des Rechts getroffen werden, möglichst als Gesetz, hilfsweise als Richterrecht.

Eine Veränderung der Werteskala zeichnet sich auch in Sachen Urheberrecht ab. Wenn die bisher fest verankerten Rechte von Künstlern und Autoren, Verlagen und Produzenten zugunsten der Internet-Allgemeinheit beiseite gedrängt werden, geht es wirklich um Wichtiges: „Tausend fundamentale Fragen, die lang beantwortet schienen, stellen sich plötzlich neu“ ${ }^{21}$ Das erste Beispiel ist „das Konzept des Eigentums“, das „durch illegale Downloads von Musik und Filmen partiell außer Kraft gesetzt worden“ sei; das werfe die Frage auf, „ob sich das Recht noch durchsetzen lässt im virtuellen Raum, der jenseits nationalstaatlicher Grenzen organisiert ist“. Wenn aber das Recht nicht mehr „vollstreckt““ werden kann - „was bleibt dann, sehr zugespitzt gesagt, vom Staat überhaupt?“

Aber nochmals: Viele dieser Fragen lassen sich mit einiger Entschiedenheit beantworten. Nur sind die Antworten selten ein pauschales Entweder/Oder, sondern meist differenzierte Lösungen, in aller Regel Kompromisse. ${ }^{22}$ Es gibt in keinem Bereich der menschlichen Gesellschaft unbegrenzte Freiheit für den Einzelnen, seine Wünsche durchzusetzen. Ein Teil der Internet-Freaks will das nicht wahrhaben und fordert eben diese Freiheit mit viel Pathos ein. Sie träumen von einer Welt, in der keine Regeln gelten (außer denen, die man sich selbst gegeben hat oder die man mit anderen vereinbart hat), daher auch niemand mit Sanktionen rechnen muss, wenn er sich nimmt, was er will. Aber auch das elektronische Netz gibt uns nicht die Chance, ein Leben zu führen, in dem die harten Realitäten der Offline-Welt ausgeblendet sind.

Zwar sind die Gedanken und Gefühle frei - aber wenn ich einen anderen Menschen beleidige oder bestehle, endet die Freiheit. Es kann dann keine Rolle spielen, ob die Grenzüberschreitung online oder offline geschieht. Lasse ich einen anderen erkennen, dass ich ihn missachte, so riskiere ich, von ihm belangt zu werden oder ihm Genugtuung verschaffen zu müssen. Wer einem anderen die Identität stiehlt oder sonstwie einen Schaden zufügt, muss damit rechnen, dass der ihn anzeigt und vor Gericht zieht. Nicht nur der Geschäftsverkehr in der Außenwelt, sondern eben auch die elektronisch vermittelten Rechtsgeschäfte können nicht „rechtsfrei“ abgewickelt werden. Mag sein, dass es angemessener ist, manche Streitigkeiten im Schiedsverfahren zu bewältigen - aber auch das ist keine Besonderheit des Internethandels; auch im traditionellen Geschäftsleben wird manch ein Prozess nicht vor staatlichen Gerichten, sondern vor vereinbarten Schiedsgerichten ausgetragen. Mag sein auch, dass manche Streitfragen gar nicht nach rechtlichen Maßstäben

21 Wefing 2011 (auch die folgenden Zitate).

22 Sehr lesenswert dazu Passig/Lobo 2012. 
entschieden werden, sondern dass es besser ist, sich mit einer moralischen Beurteilung zu begnügen - irgendwo jedoch beginnt der Bereich, in dem die moralische Verurteilung nicht mehr ausreicht, Frieden zwischen den Beteiligten herzustellen, und die Geschädigten nach harten rechtlichen Sanktionen verlangen.

\section{Die Bestimmung der Akteure, Verantwortlichen und Nutznießer}

Aufgabe des Rechts ist es auch, Akteure und damit Verantwortliche herauszuarbeiten (natürlich aufgrund politischer und medialer sozialethischer Diskussionen). Wenn nicht feststeht, welche natürliche oder juristische Person für eine Handlung oder einen Vorgang verantwortlich ist, kann kein Missstand ausgeräumt und kein Vergehen sanktioniert werden. In Teilen der Internet-Literatur scheint diese simple Erkenntnis noch nicht angekommen zu sein; sonst würden wir nicht immer wieder jene versponnenen Vorstellungen von der Wirkkraft des Netzes selbst lesen, die auf eine Verschmelzung von Menschen und Maschinen hinauslaufen und damit die Verantwortung für die Veränderung der Welt vernebeln. Der „Zukunftsforscher“ Kevin Kelly wird mit dem Satz zitiert: „Je mehr wir diesen Megacomputer benutzen, desto mehr wird er die Verantwortung für unser Wissen übernehmen. Dann wird er unser Gedächtnis. Und dann unsere Identität“. Eine Soziologin und Psychologin am berühmten MIT in Harvard namens Sherry Turkle soll erforscht haben, dass intensive Nutzer der diversen digitalen Geräte zu „einer Art Cyborg“werden, indem sie „mit der Technik in einer Weise eins“ würden, „die noch vor wenigen Jahren auch für sie selbst unvorstellbar gewesen sei““. ${ }^{23}$ Ein neues Kunstwort, dessen Bedeutung man nur erahnen kann - ist das der Stoff, aus dem wir uns ein Urteil über soziale und geistige Entwicklungen bilden können?

Nein, für rechtliche und politische Zurechnungen ist diese Art von Computerwissenschaft unbrauchbar. Sie bedeutet nämlich den Verzicht auf eine Steuerung der gesellschaftlichen Prozesse. Die Frage nach der Verantwortung wird unbeantwortbar, eine Zurechnung von Handlungen zu Personen unmöglich; wer will schon einen „Megacomputer“ oder einen „Cyborg“ korrigieren oder zur Rechenschaft ziehen? Wenn aber menschliche Verantwortung entfällt, entfallen zugleich die Vorstellungen von Gut und Böse, Recht und Unrecht. Nichts stört dann mehr die Organisatoren bei ihren Geschäften! Solche Theorien sind also nicht nur für Juristen eine Zumutung. Sie hebeln auch politische Reaktionsmöglichkeiten aus, und man fragt sich, ob das ihren Urhebern wohl bewusst ist.

Für Freiheit und Rechtsschutz im Internet ist statt dessen die Frage bedeutsam, wer das Internet und seine einzelnen Elemente betreibt, wer also als Akteur ange- 
sehen werden muss, an den sich rechtliche Anforderungen richten können. Die Staaten sind es nicht (ausgenommen sie betätigen sich als Zensoren und trennen nationale Netze von den internationalen Verbindungen!), und es gibt überhaupt keinen Alleinbetreiber oder Alleinverantwortlichen für das weltweite Netz, sondern verschiedene Netzbetreiber und zahllose Anbieter von Diensten aller Art, riesige, große und kleine Unternehmen, Einzelpersonen und Vereinigungen, seriöse und unseriöse, altruistische und raffgierige.

Entstanden ist das Internet bekanntlich aus Datenübertragungsnetzen, die das US-amerikanische Verteidigungsministerium für Forschungszwecke eingerichtet hat. ${ }^{24}$ Dabei sollte die Anfälligkeit der bis dahin bestehenden Verbindungen durch eine dezentrale Anordnung beseitigt werden; bei Störungen der einen Leitung sollte automatisch auf eine andere ausgewichen werden. Dieses Prinzip hat sich sofort bewährt und wurde für zivile Zwecke übernommen. Computerfirmen und Informationsversorger in aller Welt sahen die Chance, Transportleistungen für Datenpakete zu verkaufen - und das Ergebnis ist die globale Vernetzung aller, die es sich leisten können, vor allem der Unternehmen und Behörden, national wie international. In wenigen Jahren entstand ein dichtes Netz von Leitungen und Computern („Servern“), die den Verkehr auf den Datenleitungen steuern und gesuchte Inhalte aus den Datenmassen heraussuchen und auf die einzelnen Computer befördern.

Die Frage, wer für die im Netz gespeicherten und übermittelten Inhalte verantwortlich ist, wird damit immer schwieriger zu beantworten. Manche Beteiligten sind bloß Nutznießer, „Mitläufer“; inwieweit sie für Fehler und Rechtsverstöße verantwortlich gemacht werden können, ist zweifelhaft; andere arbeiten hochprofitabel und stehen im Zentrum des öffentlichen Interesses und der Kritik.

Ein möglicher und sinnvoller Anknüpfungspunkt für rechtliche Zurechnung ergibt sich aus der Gewinnträchtigkeit der jeweiligen Aktivtäten. Ausgleichende Gerechtigkeit verlangt, dass derjenige, der aus einer Einrichtung Vorteile zieht, auch die Nachteile tragen muss, die dadurch regelmäßig entstehen, also z.B. für Fehler haften muss. Bisher wird das Internet teilweise aus Netzanschlussentgelten finanziert, die an die Betreiber der Telekommunikationsnetze und der vermittelnden Server gezahlt werden, und in geringem Umfang auch aus zusätzlichen Gebühren, die für Sonderdienste zu entrichten sind. Die Unternehmen, die das Suchen und Befördern von Informationen anbieten, bestreiten den größten Teil ihrer Kosten aus Entgelten für die Werbeeinblendungen, die sie auf ihren Seiten platzieren, und aus der Vermarktung personenbezogener Daten über die Nutzer zum Zwecke der immer raffinierter werdenden Werbung. Das Profitstreben der Unternehmen ist zwar noch kein ausreichendes Kriterium für die Zurechnung von Rechtsverletzungen, aber wenn sie mit der Herrschaft über die Daten und der Möglichkeit zur 
Vermeidung von Beeinträchtigungen verbunden ist, spricht viel für eine rechtliche Verantwortlichkeit der Netzbetreiber oder zumindest der Diensteanbieter. Das geltende Telemediengesetz bestimmt, dass Diensteanbieter für die Speicherung, Durchleitung oder Übermittlung fremder Informationen nur ausnahmsweise verantwortlich sind, ${ }^{25}$ aber diese Regelung ist durchaus umstritten und könnte geändert werden. Es ist eine wichtige Aufgabe der Netzpolitik, in dieser Frage mehr Klarheit zu schaffen. ${ }^{26}$

Die Staaten haben ebenfalls Interessen in Bezug auf das Netz. Sie wollen an den Vorteilen des Netzes teilhaben, also selbst als Nutzer mit im Spiel sein. Andererseits müssen sie gegenüber anderen Nutzern und gegenüber den Betreibern ihre Rechtsordnung zur Geltung bringen, sofern die Nutzung in Formen geschieht, die nach der nationalen oder völkerrechtlichen Ordnung rechtswidrig sind. An der Gesamtleistung „Internet“ sind sie bisher nur marginal beteiligt, etwa durch Bereitstellen von Leitungen (in Deutschland überwiegend eine Aufgabe von Staat und Kommunen, die aber von Konzessionsnehmern wie der Deutschen Telekom ausgeführt wird). Aber das kann sich ändern. ${ }^{27}$

Die große „Gemeinde“ der Internetnutzer kann sich nur mittelbar, über die Regierungen und Parlamente der Staaten und einige supra- oder internationale Organisationen äußern. Eine Grundvoraussetzung des Internetbetriebs, die Zuordnung der Anschlüsse unter einer eindeutigen Identifizierung, also einer Nummernfolge, die niemandem sonst zugewiesen ist, wird auf der obersten Ebene, bei den übergreifenden „Domains“, von einer privaten Organisation gewährleistet. Das ist eine merkwürdige Geschichte: Das amerikanische Handelsministerium hat diese Aufgabe Mitte der 1990er Jahre an eine halbamtliche Organisation namens Internet Assigned Numbers Authority (IANA) übertragen, von der es heißt, ihr einziger Mitarbeiter sei der Internet-Pionier Jonathan Postel gewesen. 1998 wurde diese Organisation in eine andere integriert, die sich Internet Corporation for Assigned Names and Numbers (ICANN) nennt. ${ }^{28}$ Bei ICANN handelt es sich um eine Non-

25 Vgl. $\S \S 8-10$ TMG.

26 Dazu Spindler 2012, S. 60 ff. S. a. unten S. 35 mit Fn. 59. Über die Verantwortlichkeit der Internetprovider oder „Intermediären“ für Urheberrechtsverletzungen und ihre entsprechenden Pflichten zur Filterung von Inhalten wird vor nationalen und europäischen Gerichten inzwischen heftig gestritten. Der EuGH hat die geltenden EU-Richtlinien so ausgelegt, dass Anbieter von Internetzugangsdiensten nicht dazu verpflichtet werden dürfen, ein umfassendes und ,perfektes“ System der Filterung aller seine Dienste durchlaufenden elektronischen Kommunikationen einzurichten; vgl. Urteil v. 24.11.2011, JZ 2012, 308 (Scarlet ./. SABAM) m. zust. Anm. und weiteren Hinweisen von Spindler S. 311. Die Diskussion ist damit aber noch lange nicht beendet (so auch Spindler, JZ 2012, $312 \mathrm{f}$.).

27 Interessante, historisch fundierte Überlegungen dazu bei Passig/Lobo 2012, S. $177 \mathrm{ff}$.

28 Eisel 2011, S. 65; dort auch Details zur Verwaltung der deutschen Top Level Domain „de“: Die deutschen Internet Service-Provider vergaben die Domainverwaltung nach einer Ausschreibung an das Rechenzentrum der Universität Karlsruhe. Später gründeten die deutschen Service-Provider eine Genossenschaft zur Domainverwaltung, die DENIC mit Sitz in Frankfurt am Main. S.a. Ahlert, in: Holznagel/ Grünwald/Hanßmann 2001, S. 44 ff. 
Profit-Organisation, genauer eine privatrechtliche Stiftung nach US-amerikanischem Recht mit Sitz in Marina del Rey in Kalifornien. Die Regierungen sind im Governmental Advisory Committee vertreten, aber im Wesentlichen handelt es sich um eine Form von Selbstregulierung der interessierten Kreise, Unternehmen und Verbände - angesichts der Aufgabe der Organisation vielleicht eine angemessene Form. Wie diese wichtige Organisation tatsächlich funktioniert, ist für den Außenstehenden freilich schwer zu erkennen. Klar ist nur, dass auch hier Macht ausgeübt wird. Die angebliche Hierarchiefreiheit der Internet-Organisation ist eine Legende.

Ob die Regierungen sich dauerhaft zurückhalten, ist ungewiss. ${ }^{29}$ Soweit bei der Nummernvergabe wiederholt oder gar systematisch ungerecht - gleichheitswidrig und diskriminierend oder protektionistisch - vorgegangen werden sollte, werden sie sich mit Sicherheit einmischen; denn schon um seiner eigenen Legitimation willen kann kein Staat sich vor der Verantwortung drücken, wenn die privat-gesellschaftliche Organisation ihre Aufgabe verfehlt. Arme und Reiche, eigene und fremde Staatsangehörige müssen gleich behandelt werden, das lässt sich grenzüberschreitend mit Verbindlichkeit nur durch Übereinkünfte der Staaten garantieren. Auch die internationale Gerichtsbarkeit wird sich eines nicht sehr fernen Tages mit derartigen Streitfragen beschäftigen müssen.

Die offenen Flanken des Rechtsschutzes - und wie sie zu schließen sind

Ubiquität und Internationalität kennzeichnen den heutigen Stand der Datenverarbeitung: Die Computer sind überall, in miniaturisierter Form sogar in allen möglichen Gegenständen und als Herzschrittmacher oder Messgeräte im menschlichen Körper. Unzählige Computer sind über alle nationalen Grenzen und Staatenverbünde hinweg miteinander verbunden, und gewaltige Datenmengen werden irgendwo in der weiten Welt, wo gerade Platz ist, in „clouds“ gespeichert, und diese „Wolken“ kennen erst recht keine nationalen Grenzen, sondern „regnen“ ihren Inhalt mal hier, mal dort ab.

Für den Rechtsschutz hat diese Entwicklung fast unlösbare Probleme verursacht. Die Durchsetzung von Individualrechten erscheint so gut wie aussichtslos, wenn die Daten, um die es geht, nicht mehr nur einmal in einer bestimmten Datei gespeichert sind, sondern an eine Vielzahl von Adressaten übermittelt werden, und wenn jeden Augenblick neue Daten erzeugt und verarbeitet werden. Die Betroffe-

29 Es gibt bereits Bestrebungen, Aufgaben der ICANN auf die Internationale Fernmeldeunion ITU zu übertragen, also auf eine internationale Organisation, die zur UNO gehört und damit jedenfalls mittelbar dem Einfluss der Regierungen unterliegt, vgl. Küchemann 2012. Ob diese Organisationsform besser wäre, lässt sich schwer sagen. 
nen und ihre Rechtsanwälte, aber auch die Richter und Staatsanwälte müssen hinter den Akteuren herlaufen und holen sie selten ein. Der Einzelne, der sein Recht sucht, ist regelmäßig schon mit der Aufgabe überfordert, die Verantwortlichen herauszufinden, geschweige denn ein Unternehmen wie Facebook oder Google zu verklagen. Auch strafrechtlich werden Datenschutzverstöße in Deutschland bisher kaum verfolgt. ${ }^{30}$ Bei den internationalen Internetfirmen kommt zu dem normalen Prozessrisiko hinzu, dass sie nach fremdem Recht agieren und in ihren Geschäftsbedingungen einseitig bestimmen, welche Rechte und Pflichten die Vertragspartner haben - ein Machtübergewicht der Betreiber, gegen das Einzelne nur schwer ankommen können.

Gleichwohl ist es nicht vollkommen aussichtslos, individuelle Rechte auch gegen die mächtigen Unternehmen durchzusetzen. Sind andere Unternehmen betroffen, so pflegen sie ihre Interessen trotz der dargestellten Schwierigkeiten mit Nachdruck zu verfolgen; ihnen stehen hochspezialisierte Anwaltskanzleien zur Seite. Aber auch „der kleine Mann“ kann manches erreichen, wenn er hartnäckig genug ist. Der Wiener Jurastudent Max Schrems hat den Großkonzern Facebook in Verlegenheit gebracht und erste organisatorische Änderungen bei ihm provoziert, indem er schlicht Auskunft über die von ihm gespeicherten Daten verlangte: Facebook lieferte ihm eine unerwartet umfangreiche Auskunft, darunter viele Daten, die längst hätten gelöscht sein müssen und teilweise auch als gelöscht bezeichnet waren. Schrems zeigte Facebook bei der zuständigen Datenschutzaufsichtsbehörde, dem irischen Data Protection Commissioner an, u.a. wegen irreführender Geschäftsbedingungen und anderer Verstöße gegen europäisches Recht, und setzte damit Ermittlungen in Gang, die von dem Konzern offenbar mit größtem Unbehagen zur Kenntnis genommen wurden. ${ }^{31}$ Die Sache ist für die Kritiker noch lange nicht gewonnen, aber dass Facebook diese Nadelstiche ernst nimmt, zeigt sich auch darin, dass sein Europa-Bevollmächtigter Richard Allan versuchte, Schrems von einer Klage abzubringen. ${ }^{32}$ Übrigens sind inzwischen viele andere Facebook-Nutzer dem Vorbild des ,,aufmüpfigen“ Juristen gefolgt und setzen Facebook mit ihren Beschwerden zu.

Leichter wäre es für die betroffenen „Normalnutzer“, wenn es ein Verbandsklagerecht von Organisationen gäbe, die die verschiedenen Klagen und Beschwerden bündeln könnten. So wird vorgeschlagen, nach dem Vorbild des Umweltschutzes speziellen Verbänden von Datenschützern ein Klagerecht einzuräu-

30 Sieber 2012, S. 29 mit Hinweis auf die amtliche Kriminalstatistik.

31 Prummer 2012. Unter dem Titel „Europe versus Facebook“ betreibt Schrems eine Webseite mit zahlreichen Nachweisen zur Medienberichterstattung (www.europe-v-facebook.org). Der Bericht der irischen Behörde Data Protection Commissioner: Facebook Ireland Ltd. Report of the Re-Audit, 21 September 2012) ist zugänglich unter www.dataprotection.ie . Danach muss Facebook nunmehr z.B. die Gesichtserkennung abschalten.

32 Prummer 2012. 
men. ${ }^{33}$ Die Verbandsklage ist jetzt auch in dem Entwurf der Europäischen Datenschutz-Grundverordnung vorgesehen. ${ }^{34}$ Damit wird an die amerikanische Methode angeknüpft, in „Sammelklagen“ (,class actions“) den Rechtsschutz gegen solche Verstöße zu bündeln, die für sich genommen einen Prozess nicht lohnen. Diese Konstruktion würde einen neuen Markt für Juristen eröffnen - vermutlich mit ähnlichen Folgen wie derzeit bei Urheberrechtsstreitigkeiten, dass nämlich Spezialisten für „Abmahnungen“ damit gute Geschäfte machen. Die Rechtsentwicklung aber könnte dadurch vorangetrieben werden; denn auf diese Weise würden aus vielen kleinen Fällen neue Regeln entwickelt - Richterrecht, das konkreter und sachnäher sein kann als die allgemein gehaltenen gesetzlichen Vorschriften.

Ein anderer Vorschlag zielt darauf ab, ,grenzüberschreitende onlinespezifische Schiedsverfahren“ einzurichten. ${ }^{35}$ Auch damit könnte einiges bewirkt werden freilich wohl in erster Linie zwischen hinreichend großen, organisationsstarken Unternehmen und Verbänden. ${ }^{36}$ Microsoft, Google und Facebook mögen auf diesem Wege ihre Rechtsstreitigkeiten bewältigen und neue verbindliche Regeln produzieren, aber es dürfte schwer sein, die Betroffenen in diese Schiedsverfahren einzubeziehen.

Die Grenzen der nationalen Rechtsordnung ${ }^{37}$ können jedenfalls in der Europäischen Union überwunden werden, indem gemeinsames Recht geschaffen wird. Das wird gegenwärtig versucht: Es wird intensiv über den erwähnten Entwurf einer Datenschutz-Grundverordnung beraten. Die damit intendierte umfassende und intensive Regelung des Datenschutzes stößt allerdings auf Bedenken: Diejenigen EU-Mitgliedstaaten, die wie die Bundesrepublik ein hohes Datenschutzniveau haben, ${ }^{38}$ wollen darauf nicht verzichten; ein Richter des Bundesverfassungsgerichts sieht sogar die weltweit beachtete Rechtsprechung dieses Gerichts und die Geltung unserer Grundrechte in der Gefahr, einer Nivellierung auf europäisches Mittelmaß zum Opfer zu fallen. ${ }^{39}$ Andererseits wird es wohl kaum eine europäische Rechtsharmonisierung geben, wenn für alle Mitgliedstaaten der höchste denkbare Standard gefordert wird.

Der Europarat hat vor einigen Jahren eine „Cybercrime-Konvention“ beschlossen, ${ }^{40}$ die eine Vereinheitlichung der in Betracht kommenden Straftatbestände verlangt, aber keine spezifischen Regelungen zur Gerichtsbarkeit enthält. Es gibt auch

33 S. etwa Spindler 2012, S. 130 f. m.w.N.

34 Vgl. Europäische Kommission 2012, Art. 75 Abs. 2 und Art. 76 Abs. 1 des Entwurfs.

35 Spindler 2012, S. 113 f. und These 11, S. 134.

36 S. aber auch den Vorschlag von Ladeur 2012, unabhängige „Cyber-Courts“ einzurichten; dazu noch unten S. $124 \mathrm{f}$.

37 Dazu grundsätzlich und ausführlich Determann 1999. S. a. Franda 2001.

38 Schaar 2007, S. 26 mit Hinweisen auf ,internationale Hitlisten“.

39 Masing $2012 \mathrm{a}$.

40 Vgl. Sieber 2012, S. 41 und 75. 
Initiativen der EU über die geplante Datenschutz-Grundverordnung hinaus, und es gibt Forderungen nach einer UN-Konvention. ${ }^{41}$

Selbst internationale Rechtshilfe läuft ins Leere, wenn die einschlägigen Rechtsvorschriften die Anwendung einer Rechtsordnung gebieten, die dem Betroffenen im konkreten Fall keine ausreichenden Rechte zuweist. Das geschieht häufig, wenn Europäer versuchen, Verstöße gegen ihre Vorstellungen von Datenschutz in angelsächsischen Ländern zu verfolgen. So „ordnet das Recht der Vereinigten Staaten von Amerika den Datenschutz über ein Flickwerk von Regeln, die große Bereiche frei von jeder formellen Regulierung lassen ". ${ }^{42}$ Berichtet wird von einem deutschen Richter, ,der Zugriff auf Facebookdaten suchte, dann auf die Facebook-Niederlassung in Irland verwiesen wurde und diese schließlich erklärte, dass die Daten leider auf Servern in den USA lägen" “. ${ }^{43}$ Dieser Fall macht übrigens deutlich, dass es nicht sachgerecht ist, an den Ort der Datenspeicherung und damit den Sitz des Anbieters anzuknüpfen; die Rechtsverfolgung muss vielmehr dort möglich sein, wo der Nutzer seinen Sitz hat - die Experten sprechen vom „Marktortprinzip“. ${ }^{4}$

Wenn die Europäische Datenschutz-Grundverordnung verbindlich wird, ergibt sich innerhalb der Union eine weitgehend einheitliche Rechtslage. Aber jeder, der einmal mit der Anwendung unbestimmter Rechtsbegriffe zu tun hatte, weiß um die Schwierigkeiten, solche Normen einheitlich anzuwenden. Enthält eine Vorschrift Begriffe wie „öffentliches Interesse“ oder „Wesensgehalt des Rechts auf den Schutz personenbezogener Daten“, so sind ganz unterschiedliche Interpretationen und folglich eine divergierende Anwendung der Norm unvermeidlich. Die zitierten und ähnlich weite Formulierungen stehen in der EU-Grundverordnung an mehreren zentralen Stellen. ${ }^{45}$ Immer wieder bedingen Datenschutzvorschriften des nationalen und supranationalen Rechts eine Abwägung zwischen verschiedenen Grundrechten oder Interessen, ohne dass dazu Richtlinien gegeben würden - auch dies eine Quelle ständiger Meinungsverschiedenheiten.

Deren Lösung erfordert Zuständigkeitsregeln, die einer der beteiligten Instanzen die verbindliche Entscheidung zuweist. Die EU-Verordnung versucht dies zunächst, indem sie die nationalen Aufsichtsbehörden zur gegenseitigen Amtshilfe verpflichtet und ihnen gemeinsame Maßnahmen gestattet und vor allem ein geregeltes „Kohärenzverfahren“ einführt. ${ }^{46}$ Zusätzlich wird ein unabhängiger Europäischer Datenschutzausschuss eingerichtet, der durch Beratung der Kommission

41 So etwa Dix, in: DJT 2012, S. 73 These 19.

42 Paul M. Schwartz, in: DJT 2012, S. 75 These 2.

43 Masing 2012 b, S. 2310 m.w.N.

44 Osthaus, in: DJT 2012, S. 75 These 15; Spindler ebd. S. 68 These 14.

45 Vgl. Europäische Kommission 2012, Art. 6 Abs. 3 (betrifft die Rechtmäßigkeit der Datenverarbeitung) und Art. 17 Abs. 3 Buchstabe d (betrifft Ausnahmen von der Pflicht zur Löschung von Daten). Auf das „öffentliche Interesse“ stellen noch weitere Normen der EGGrundverordnung ab.

46 Art. 55-63 des Entwurfs. 
und Stellungnahmen zu Beschlussentwürfen im Kohärenzverfahren die einheitliche Anwendung der Verordnung „,sicherstellen“ soll. ${ }^{47}$ Bei wesentlichen Streitfragen wird ihm dies aber wohl kaum gelingen. Zwar kann mit den bezeichneten Vorkehrungen gewiss ein hohes $\mathrm{Ma} ß$ an Einheitlichkeit erreicht werden, aber eben keine verbindliche Letztentscheidung. ${ }^{48}$ In einigen besonderen Fällen kann die Kommission „Durchführungsrechtsakte“ erlassen, ${ }^{49}$ aber im Prinzip behalten die nationalen Aufsichtsbehörden die Verantwortung für ihre Entscheidungen, und die Betroffenen müssen sich um Rechtsschutz vor den nationalen Gerichten bemühen. ${ }^{50}$

Einen weiteren Ansatz der Vereinheitlichung bildet jedoch die vorgesehene Befugnis der Kommission, „delegierte Rechtsakte“ zu erlassen, die der Konkretisierung der unbestimmten Formulierungen dienen sollen. ${ }^{51}$ Auch darüber wird es noch viel Streit geben; denn die Kommission muss dann all die konkreten Abwägungen nachholen, die vor dem Entwurf der Verordnung versäumt worden sind.

Aber nochmals: Der Versuch einer Vereinheitlichung ist richtig, die einzelnen Instrumente dafür müssen diskutiert werden, und auf der Ebene der Vereinten Nationen sind weitere Schritte nötig. Denn es ist ja Konsens, dass die Menschen auf der ganzen Welt vor den Risiken der Informationstechnik geschützt werden müssen. Ohne verpflichtendes Recht ist dies unmöglich.

47 Art. 64-72, Zitat: Art. 66 Abs. 1 Satz 1.

48 Daher fordert Dix (in: DJT 2012, S. 73 These 20) das Letztentscheidungsrecht des Europäischen Datenschutzausschusses.

49 Art. 62.

50 Art. 74 und 75.

51 Vgl. Art. 86 mit der langen Liste der Einzelermächtigungen! Weiteres s. unten S. 142 f. 